\title{
SHORT COMMUNICATION CpG-based immunotherapy impairs antitumor activity of BRAF inhibitors in a B-cell-dependent manner
}

\author{
L Huang ${ }^{1}$, Z Wang ${ }^{1}$, C Liu, C Xu, RM Mbofung, JA McKenzie, H Khong, P Hwu and W Peng
}

Combining immunotherapy with targeted therapy has increasingly become an appealing therapeutic paradigm for cancer treatment due to its great potential for generating durable and synergistic antitumor response. In this study, however, we unexpectedly found that two types of CpG-based tumor peptide vaccine treatments consistently negated the antitumor activity of a selective BRAF inhibitor in tumors with BRAF mutation rather than showing a synergistic antitumor effect. Our further studies demonstrated that $\mathrm{CpG}$ alone was sufficient to dampen BRAF inhibitor-induced antitumor responses, suggesting that the impaired antitumor activity of the BRAF inhibitor observed in mice receiving CpG-based peptide vaccine is mainly dependent upon the use of CpG. Mechanistically, CpG increased the number of circulating B cells, which produced elevated amounts of tumor necrosis factor-a (TNFa) that contributed to the increased tumor resistance to BRAF inhibitors. More importantly, B-cell depletion or TNFa neutralization can restore the antitumor effect of BRAF inhibition in mice receiving $\mathrm{CpG}$ treatment, indicating that TNFa-secreting $B$ cells play an indispensable role in BRAF inhibitor resistance induced by $\mathrm{CpG}$. Taken together, our results strongly suggest that precautions must be implemented when designing combinatorial approaches for cancer treatment, because distinct regimens, despite their respective therapeutic benefit as monotherapy, may together provide antagonistic clinical outcomes.

Oncogene (2017) 36, 4081-4086; doi:10.1038/onc.2017.35; published online 6 March 2017

\section{INTRODUCTION}

Combining targeted therapy with immunotherapy has increasingly become an appealing therapeutic strategy for cancer treatment due to its great potential for improved overall efficacy and durable antitumor response. ${ }^{1,2}$ Indeed, using animal models and patient samples, we and others have demonstrated a synergistic outcome of combining targeted therapy with immunotherapy. ${ }^{3-6}$ For example, co-administration of a selective BRAF inhibitor with adoptively transferred T lymphocytes results in significantly enhanced tumor control in BRAF-mutant melanoma. ${ }^{6}$ Additionally, immune checkpoint blockade using anti-PD1 antibodies synergizes with BRAF inhibition, providing increased therapeutic benefit when compared with the targeted therapy alone. ${ }^{3}$ It is not known whether targeted inhibition of BRAF synergizes with other immunotherapeutic strategies, such as cancer vaccines, which would generate de novo anti-cancer immune responses. ${ }^{7,8}$

Among various regimens of cancer vaccines that have been designed and shown to benefit cancer patients, administration of peptides harboring tumor-specific T-cell epitopes may represent a convenient vaccination strategy due to the ease of peptide synthesis and purification. Adjuvants are often used together with these peptides to stimulate the immune response to the antigen. CpG oligodeoxynucleotides (CpG-ODN), short single-stranded synthetic DNA molecules containing unmethylated cytosineguanine motifs, represent one type of the widely employed vaccine adjuvants due to its potency in promoting antigenspecific immune responses. ${ }^{9,10} \mathrm{CpG}$ motifs are abundant in microbial genomes but rare in vertebrate genomes, and thus are considered as pathogen-associated molecular patterns. ${ }^{11}$ They can be recognized by the pattern recognition receptor, Toll-like receptor 9, which is constitutively expressed on B cells and plasmacytoid dendritic cells in humans and rodents. ${ }^{12}$ Therefore, CpG can activate Toll-like receptor 9 on B cells and plasmacytoid dendritic cells and thereby regulate subsequent immune response to vaccines. Notably, in multiple murine tumor models, CpG adjuvants are essential for inducing activation and accumulation of cytotoxic T lymphocytes that are specific to tumor antigens. ${ }^{13,14}$

There are five classes of CpG ODNs (Class A, Class B, Class C, Class $P$ and Class $S$ ) based on their sequences and secondary structures. ${ }^{15}$ Among these, Class A and Class B CpG ODNs are the most frequently used adjuvants to treat patients with melanoma, lung, ovarian, breast and colon cancers, ${ }^{16}$ and display ability to enhance T-cell-mediated antitumor response. In a clinical study, melanoma patients treated with CpG-based peptide vaccines demonstrated increased amounts of circulating Melan-A-specific $\mathrm{CD}^{+} \mathrm{T}$ cells when compared with treatment without CpG adjuvants. ${ }^{17}$ Based upon these previous observations, we hypothesize that combination of CpG-based peptide vaccines and BRAF inhibitors can generate synergistic antitumor effects. Unexpectedly, however, our results showed that using CpG as the vaccine adjuvant impaired the antitumor activity of BRAF inhibitors in mouse models of BRAF-mutant melanoma, and this depends on increased levels of tumor necrosis factor-a (TNFa) produced by $B$ cells. These results strongly suggest that precautions must be taken when combining targeted therapy

Department of Melanoma Medical Oncology, University of Texas MD Anderson Cancer Center, Houston, TX, USA. Correspondence: Dr W Peng, University of Texas MD Anderson

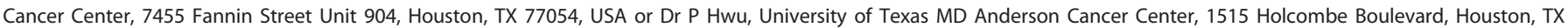
77030, USA.

E-mail: wpeng@mdanderson.org or phwu@mdanderson.org

${ }^{1}$ These two authors contributed equally to this work.

Received 2 September 2016; revised 4 January 2017; accepted 5 January 2017; published online 6 March 2017 
a

gp100 peptide+CpG (vaccine site) treatment

PLX treatment

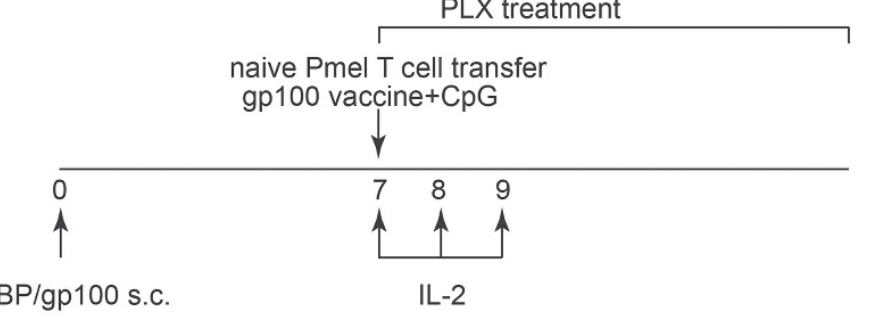

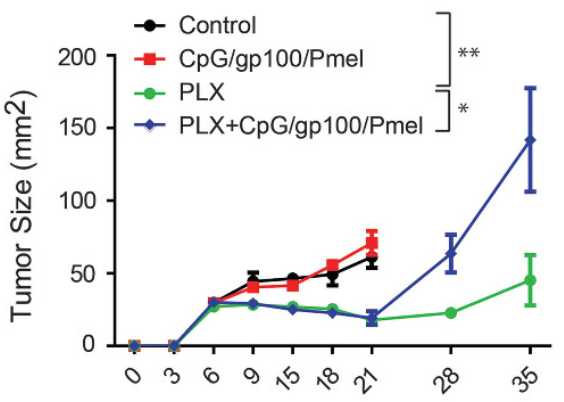

b

p15E peptide+CpG (vaccine site) treatment
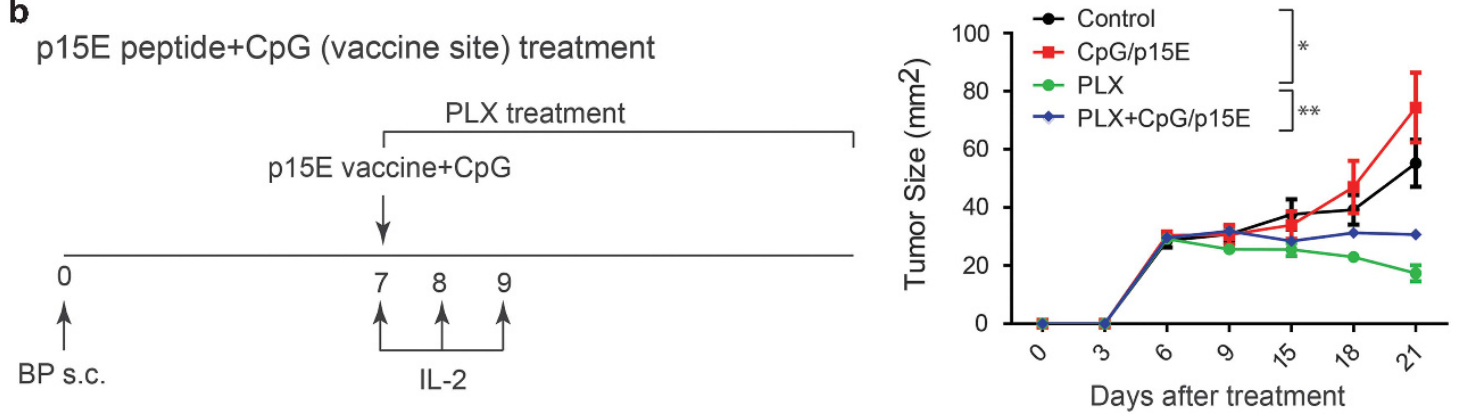

Figure 1. BRAF inhibitor-induced antitumor responses are impaired when combined with CpG-based peptide vaccines. (a) A previously established murine melanoma cell line bearing BRAF and PTEN mutation (BP) was provided by Dr Wargo (MD Anderson Cancer Center) ${ }^{3}$ and maintained in RPMI 1640 with 10\% FCS and $100 \mu \mathrm{g} / \mathrm{ml}$ Normocin (Invivogen, San Diego, CA, USA). To generate gp100-expressing murine cell line (BP/gp100), BP cells were transduced with the lentiviral vector encoding full-length human gp100 as previously described. ${ }^{34}$ Tumor growth was induced by subcutaneous injection of $5 \times 10^{5} \mathrm{BP} / \mathrm{gp} 100$ cells in 6- to 8-week-old female C57BL/6 mice (from Charles River NCl, Frederick, MD, USA). Seven days after tumor challenge, tumor-bearing mice were treated with a selective BRAF inhibitor PLX4720 (Plexxikon, Berkeley, CA, USA; 100 mg/kg in 3\% DMSO and $1 \%$ methylcellulose by oral gavage daily ${ }^{35}$ ), tumor vaccine with $50 \mu \mathrm{g} \mathrm{CpG} \mathrm{(CpG-ODN-2216} \mathrm{synthesized} \mathrm{by} \mathrm{Invitrogen} \mathrm{Life} \mathrm{Technologies}$ (Carlsbad, CA, USA) and injected intratumorally once per week for 3 weeks) and $100 \mu \mathrm{g}$ synthetic, high-affinity $\mathrm{H}_{-2} \mathrm{D}^{\mathrm{b}}$-restricted hgp $10 \mathrm{O}_{25}-33$ peptide (KVPRNQDWL, purchased from Peptides International (Louisville, KY, USA) at a purity $>95 \%$ and injected subcutaneously), or both. For groups containing the tumor vaccine, $1 \times 10^{3}$ Pmel-1T cells (isolated from Pmel-1 TCR/Thy1.1 mice from in-house breeding colonies) were intravenously administered and $100 \mu \mathrm{g}$ anti-CD40 (Bioxcell, West Lebanon, NH, USA) was intraperitoneally injected on day 7 , and $1 \times 10^{5} \mathrm{IU}$ rhIL-2 protein (Prometheus Laboratories Inc, San Diego, CA, USA) were intraperitoneally injected on days 7, 8 and 9. (b) Tumor growth was induced by subcutaneous injection of $5 \times 10^{5}$ BP cells in 6- to 8-week-old female C57BL/6 mice. Seven days after tumor challenge, tumor-bearing mice were treated with PLX4720, tumor vaccine with $50 \mu \mathrm{g} \mathrm{CpG}$ and $100 \mu \mathrm{g} \mathrm{H}-2 \mathrm{~K}^{\mathrm{b}-}$ restricted mouse P15E $134-141$ peptide (KSPWFTTL, purchased from Peptides International at a purity $>95 \%$ and injected subcutaneously), or both. For groups containing the tumor vaccine, $100 \mu \mathrm{g}$ anti-CD40 was intraperitoneally injected on day 7 , and $1 \times 10^{5} \mathrm{IU}$ rhlL-2 protein were intraperitoneally injected on days 7,8 and 9 . Tumor-bearing mice treated with vehicle (3\% DMSO and 1\% methylcellulose) were used as control. Tumor growth was monitored every 3 days by measuring the perpendicular diameters of tumors. $N=5$ mice per group. Data expressed as mean \pm s.e.m. ${ }^{*} P<0.05,{ }^{* *} P<0.01$, two-way ANOVA plus post hoc Turkey test.

and immunotherapy because these two distinct regimens, despite their respective therapeutic benefits, may together provide antagonistic, rather than synergistic, clinical outcomes.

\section{RESULTS AND DISCUSSION}

CpG-based tumor vaccines inhibit in vivo antitumor activity of BRAF inhibitors

FDA-approved BRAF inhibitors have become the frontline treatment option for melanoma patients harboring BRAF mutations. To develop and evaluate novel combinatorial therapies, we set out to determine the in vivo therapeutic effect of combining cancer vaccines with a selective BRAF inhibitor, PLX4720 (PLX). We employed two well-established models of tumor vaccines: (1) gp100 peptide vaccination plus adoptive transfer of gp100specific T cells from Pmel-1 mice ${ }^{4}$ and (2) p15E peptide vaccination. ${ }^{18}$ These different vaccination regimens focused on distinct antigenic peptides and induced exogenous and endogenous antitumor responses, respectively. CpG-ODN-2216, which displays a great potency to induce T-cell mediated antitumor immune response in murine vaccination models, ${ }^{18,19}$ was included in both vaccination approaches as the adjuvant. A spontaneous melanoma cell line established from Tyr:CreER;BRAF ${ }^{V 600 E /+} ;$ PTEN $^{\text {lox/ }}$ ${ }^{l o x}$ mice (designated as BP hereafter) mice was utilized for tumor growth studies in a syngeneic transplantable mouse model. ${ }^{3}$ Specifically, BP tumor cells stably expressing the melanoma antigen gp100 (BP/gp100) were generated and subcutaneously implanted into mice and allowed to grow for 7 days before treatment with PLX, tumor vaccines or both. Our results demonstrated that BRAF inhibition alone resulted in significantly impaired tumor growth when compared with vehicle control (Figure 1a). However, when combined with the vaccination regimen comprising gp100 peptide, CpG adjuvant, adoptively transferred Pmel-1 T cells, anti-CD40 and IL-2, the BRAF inhibitorinduced antitumor responses appeared to be significantly weakened (Figure 1a). Similarly, reduced antitumor activity from combined CpG-based vaccines and BRAF inhibitors was also observed when the second vaccination regimen targeting the endogenously expressed antigen P15E was used (Figure 1b). Together, these results indicated that, regardless of the type of antigen or the involvement of T-cell transfer, peptide-based vaccines with $\mathrm{CpG}$ adjuvant consistently impaired the tumorregressing capacity of BRAF inhibitors.

The antagonism between BRAF-targeted therapy and CpGbased tumor vaccines was an unexpected and intriguing finding. When administered as a single agent, BRAF inhibitors ${ }^{20}$ or CpG vaccination with melanoma antigens ${ }^{17,21}$ can induce tumor regression in animal studies and patient cohorts. However, our results demonstrated that when implemented as a combination 
a

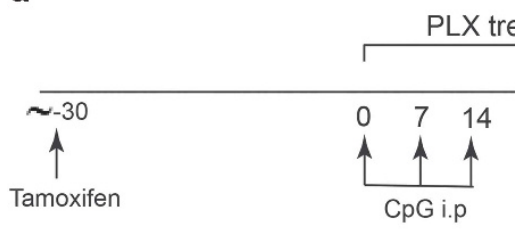

C

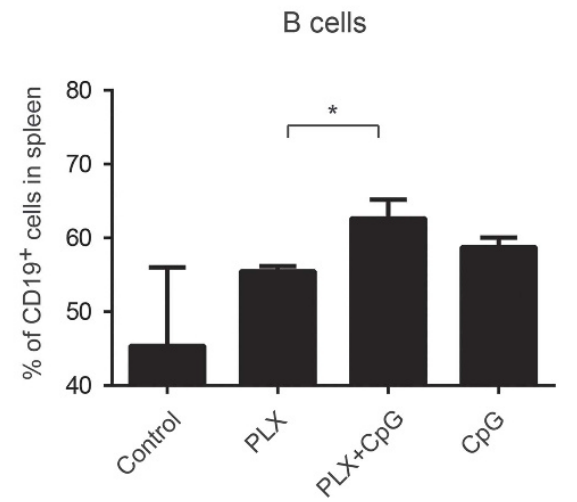

b $\rightarrow$ Control

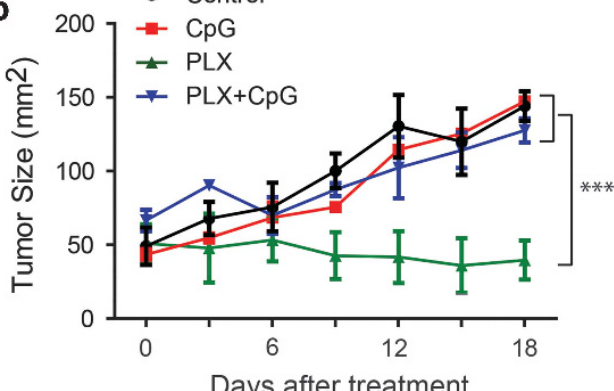

Days after treatment
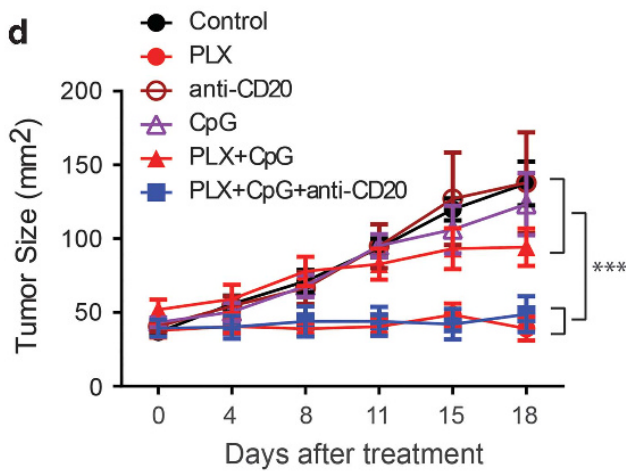

Figure 2. CPG negates the antitumor activity of BRAF inhibitors in a B-cell-dependent manner. To induce spontaneous melanoma in genetically modified mice, Tyr:CreER; PTEN ${ }^{\text {lox/lox}} ; \mathrm{BRAF}^{\mathrm{V} 600 \mathrm{E} /+}(\mathrm{BP})$ mice on a C57BL/6 background (6-8 weeks of age) were treated with $50 \mathrm{mg}^{\mathrm{ml}}{ }^{-1}$ 4-hydroxytamoxifen (Sigma, St Louis, MO, USA) to induce the expression of Cre as previously described. ${ }^{23}$ Mice with measurable tumors received indicated antitumor treatments. (a, b) Tumor-bearing BP mice were treated with daily oral gavage of $100 \mathrm{mg} / \mathrm{kg}$ PLX4720 (PLX), intraperitoneal injection of $50 \mu \mathrm{g} \mathrm{CpG}$ once per week for 3 weeks, or both. Tumor growth was monitored every 3 days by measuring the perpendicular diameters of tumors. $N=5$ mice per group. (c) Single-cell suspensions were prepared from the spleens of tumor-bearing BP mice treated with PLX, CpG or both, and stained with anti-CD4, CD8 and CD19 antibodies (BD Biosciences, San Jose, CA, USA). The percentages of various immune cell populations ( $T$ cells and B cells) in stained samples were analyzed by flow cytometry using a FACSCANTO II cell analyzer (BD Biosciences, Franklin Lakes, NJ, USA). $\mathrm{CD} 19^{+} \mathrm{B}$ cells in spleen in each group were shown. $N=5$ per group. (d) Tumor-bearing BP mice were simultaneously treated with PLX, CpG or both, as described in (a, b). Also, $50 \mu \mathrm{g}$ of anti-mouse CD20 Ab lgG2a kindly provided by Biogen Idec (San Diego, CA, USA) were injected intravenously in tumor-bearing BP mice once per week for 2 weeks to deplete B cells either alone or together with PLX and CpG. Isotype control antibody purchased from Sigma were similarly injected as a control. Tumor sizes were monitored every 3 days (Control group: $N=7 ;$ PLX group: $N=6$; anti-CD20 group: $N=5$; CpG group: $N=4$; PLX+CpG group: $N=6$; PLX+CpG+anti-CD20 group: $N=6$ ). Data expressed as mean \pm s.e.m. ${ }^{*} P<0.05$, ${ }^{* * *} P<0.001$, twoway ANOVA (b, d) or one-way ANOVA (c) plus post hoc Turkey test.

therapy, CpG-based vaccines resulted in diminished BRAF inhibitor-mediated antitumor activity. Although BRAF inhibition can synergistically improve therapeutic capacity of several types of immunotherapy, such as adoptive T-cell transfer ${ }^{5,6}$ and immune checkpoint blockade, ${ }^{3,22}$ our seemingly counterintuitive results suggest that combining targeted therapy with immunotherapy may not always generate synergistic outcomes.

CpG negates the antitumor activity of BRAF inhibitors in a B-cell-dependent manner

The various components in the tumor vaccine may be responsible for the deficient antitumor activity when combined with BRAF inhibition. However, we reasoned that $\mathrm{CpG}$ adjuvant was the most likely candidate, because the adverse impact of the vaccine appeared to be antigen-independent and regimen-irrelevant based upon results from the two distinct vaccination models. To test our hypothesis, we administered CPG intraperitoneally in combination with PLX (Figure 2a) and assessed antitumor responses in BP transgenic mice, a tamoxifen-inducible spontaneous mouse model that physiologically recapitulates the development of human melanoma. ${ }^{23}$ Our results confirmed that systemic administration of CpG alone was sufficient to dampen BRAF inhibitor-induced antitumor responses (Figure 2b), suggesting that $\mathrm{CpG}$ is largely responsible for the differential antitumor activity observed between the BRAF inhibitor alone and the combination therapy.

We next sought to determine the mechanism that underlies the adverse effect of CpG on the combination therapy. CpG may have an effect on different types of cells, including tumor cells, dendritic cells and T cells. Thus, it is possible that CpG directly changes tumor cell biology and function, which in turn may impair the antitumor effect induced by the BRAF inhibitor. To test this possibility, we treated BP tumor cells in vitro with or without $\mathrm{CpG}$ for $24 \mathrm{~h}$ and measured cell growth by CellTiter Blue assay. As shown in Supplementary Figure S1, both high and low concentrations of $\mathrm{CpG}$ failed to alter the growth of tumor cells, suggesting that the antagonistic effect of $\mathrm{CpG}$ in combination with the BRAF inhibitor could not be explained by the direct effect of CpG on tumor cells.

Given its potent immunomodulatory function, ${ }^{24} \mathrm{CpG}$ likely alters immune factors that influence the therapeutic response to BRAF inhibitors. Indeed, flow cytometry analysis revealed that the percentage of splenic B cells was significantly increased in mice treated with the combination therapy when compared with the cohorts treated with the BRAF inhibitor alone (Figure 2c). To characterize the functional relevance of the increased number of $B$ cells induced by CpG, we administered an anti-CD20 antibody intraperitoneally to deplete B cells in vivo and assessed how this impacted the role of $\mathrm{CpG}$ in the combination therapy. Upon 

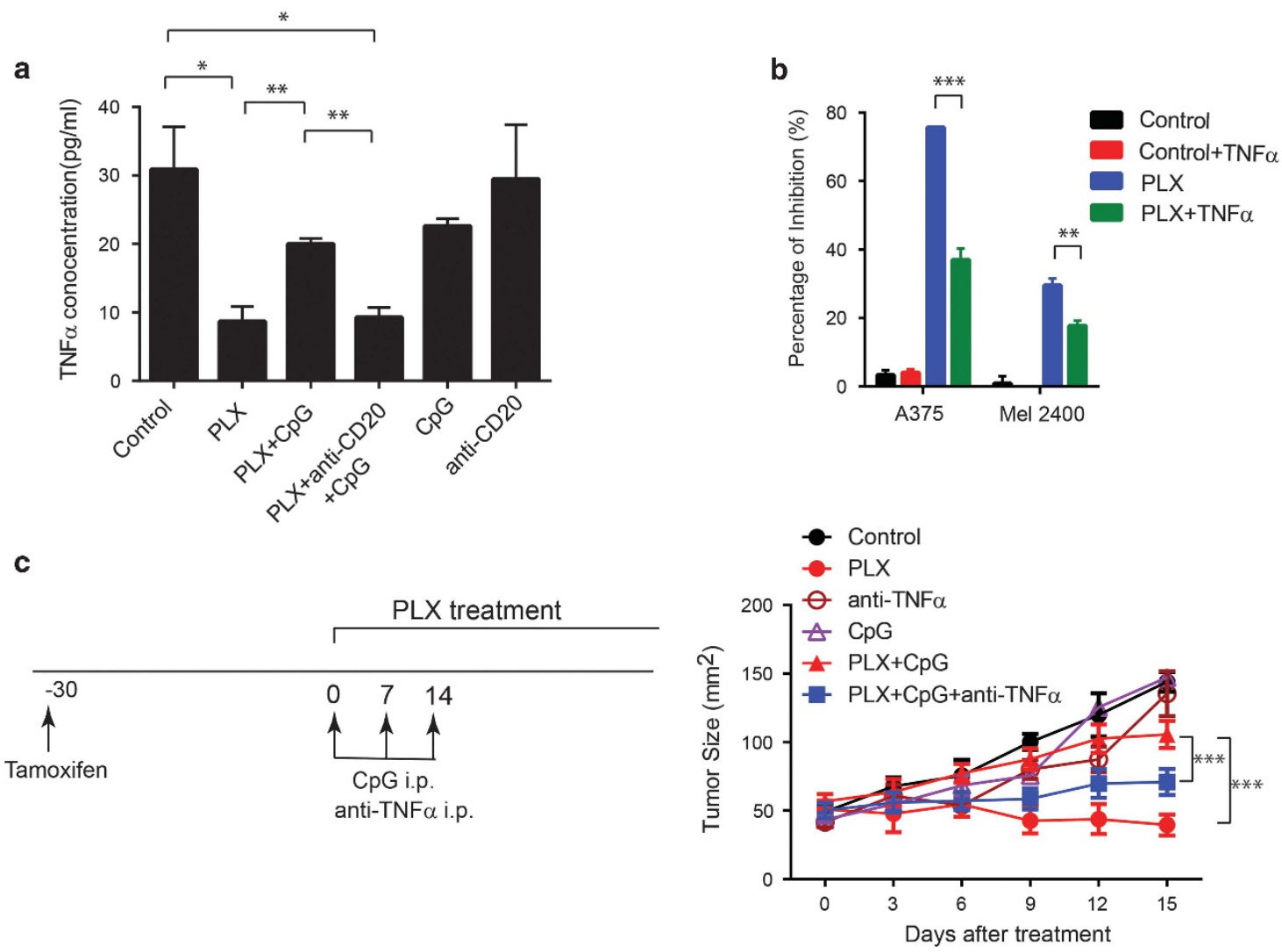

Figure 3. CpG treatment dampens BRAF inhibitor-induced tumor regression through B-cell-derived TNF $\alpha$. (a) Sera samples from tumorbearing BP mice treated as in Figure $2 \mathrm{~d}$ were collected and measured by MILLIPLEX mouse cytokine/chemokine panels I (premixed 32-plex) and II (premixed 8-plex) according to the manufacturer's protocol (EMD Millipore, Billerica, MA, USA). The concentration of each cytokine and chemokine in serum was determined using a Luminex 200 system (Luminex Corporation, Austin, TX, USA). Serum expression of TNF $\alpha$ from different treatment groups are shown. $N=3$ per group. (b) Melanoma tumor cells A375 and 2400 were seeded at 1000 cells per well and stimulated with $1 \mu \mathrm{m}$ PLX, $20 \mathrm{ng} / \mathrm{ml}$ TNF $\alpha$ (Bioxcell) or both. Three days later, cell viability was determined using CellTiter Blue assay according to the manufacturer's protocol (Promega, Madison, WI, USA). Data expressed as mean \pm s.e.m. of triplicates from two independent experiments. (c) Tumor-bearing BP mice were treated with vehicle, PLX, CpG, or combination of PLX and CpG as described in Figure 2. To neutralize TNF $\alpha$, mice were injected with $100 \mu \mathrm{g}$ of rat anti-mouse TNF $\alpha$ lgG1 (Bioxcell) intraperitoneally once per week for 2 weeks, either alone or together with PLX and CpG. Tumor sizes were monitored every 3 days. (Control group: $N=5 ; \mathrm{PLX}$ group: $N=4 ;$ anti-TNF $\alpha$ group: $N=5$; CpG group: $N=4$; PLX+CpG group: $N=6$; PLX+CpG+anti-TNF $\alpha$ group: $N=6$ ). Data expressed as mean \pm s.e.m. ${ }^{*} P<0.05$, ${ }^{* *} P<0.01$, ${ }_{* * *} P<0.001$, one-way ANOVA $(\mathbf{a}, \mathbf{b})$ or two-way ANOVA (c) plus post hoc Turkey test.

anti-CD20 administration, the percentage of $\mathrm{CD} 19^{+} \mathrm{B}$ cells was significantly reduced in blood, spleen and tumor, whereas that of $\mathrm{CD}^{+}$and $\mathrm{CD}^{+} \mathrm{T}$ cells, Gr- ${ }^{+}$granulocytes and $\mathrm{CD} 11 \mathrm{~b}^{+}$ monocytes were unaltered (Supplementary Figures S2a and b). As illustrated in Figure 2d, tumor growth in BP mice was effectively suppressed by BRAF inhibitor treatment, but not in the presence of systemic CpG administration. However, the antitumor response was restored upon B-cell depletion by anti-CD20 antibody. These data indicate that $B$ cells play an indispensable role in the resistance to BRAF inhibitors in the presence of systemic CpG treatment.

B-cell-derived TNFa contributes to CpG-induced resistance to BRAF inhibition.

To mechanistically identify how CpG-induced B cells augment resistance to BRAF inhibitors, we further characterized the profile of serum cytokines in treated mice through a Luminex multiplex assay. Among the assessed cytokines, the expression pattern of TNFa was identified to correlate with the magnitude of antitumor responses upon different types of treatment. Specifically, treatment with the BRAF inhibitor PLX resulted in significantly decreased levels of serum TNFa, which was upregulated when combined with CpG treatment (Figure 3a). Importantly, addition of a B-cell-depleting anti-CD20 antibody impeded the increase in TNFa observed with CpG treatment (Figure 3a), implicating B cells as a major source of TNFa. We further confirmed that treatment of purified $B$ cells with $\mathrm{CpG}$ in vitro induced the production of TNFa (Supplementary Figure S3a). In addition, mice injected with CpG had higher TNFa in splenic CD19 ${ }^{+}$B cells than in those without CpG treatment (Supplementary Figure S3b). Furthermore, in a transplantable model of BP tumors, treatment of tumor-bearing mice with $\mathrm{CpG}$-based vaccine dramatically increased the percentage of $\mathrm{TNFa}^{+}$cells in the $\mathrm{CD} 19^{+}$B-cell population in the spleen (Supplementary Figure S4), indicating that CpG-based vaccine can also increase TNFa-producing B cells in the spleen. These results are consistent with previous reports showing CpG-induced TNFa production from B cells. ${ }^{25,26}$ In line with previous studies demonstrating an essential role for TNFa in the resistance to mitogen-activated protein kinase signaling inhibition, ${ }^{27-29}$ our in vitro assays in two patient-derived melanoma cell lines showed that the inhibition of tumor cell growth by the BRAF inhibitor was significantly mitigated when combined with TNFa (Figure 3b). More importantly, upon administration of a neutralizing anti-TNFa antibody in tumor-bearing BP mice, inhibition of in vivo tumor growth was largely restored as compared with the combined PLX and CpG treatment (Figure 3c). Taken together, these results 
suggest that B-cell-secreted TNFa is an essential mediator for the CpG-induced resistance to BRAF inhibitor therapy.

Previous studies have shown that B cells, but not T cells, express Toll-like receptor 9 and can thus be specifically activated by $\mathrm{CpG}^{24,30}$ which is consistent with the increased production of B-cell-derived TNFa upon systemic CpG treatment observed in our study. Notably, multiple lines of evidence have implicated an important cancer-promoting role for B lymphocytes through various immunoregulatory mechanisms, potentially involving downregulated NK cell activity, less intratumoral $\mathrm{CD}^{+} \mathrm{T}$ cells and increased function of regulatory T cells. ${ }^{31-33}$ Our results added B-cell-derived TNFa as another possible layer of immunomodulatory mechanisms, because co-treatment with anti-TNFa antibodies partially rescued the impaired antitumor activity in response to the combinatorial therapy. TNFa is known to suppress apoptosis and promote growth in melanoma, thereby inducing resistance to mitogen-activated protein kinases pathway inhibitors, including those targeting BRAF. ${ }^{27-29}$ These observations suggest that better antitumor therapeutic outcomes may be achieved by simultaneously disrupting TNFa function or its downstream nuclear factor-KB pathways with targeted therapy or combinatorial immunotherapy. However, it needs to be noted that, in addition to TNFa, other B-cell-derived factors may also be accountable, because tumor resistance to the combined $\mathrm{CpG}$ and BRAF inhibitor treatment was not completely alleviated upon TNFa neutralization.

Among many currently available treatment approaches, targeted therapy and immunotherapy have demonstrated impressive clinical benefit for cancer patients. To seek even better antitumor responses, recent studies have exploited the possibility of combining targeted therapy and immunotherapy, which have shown improved therapeutic outcomes in animal models and clinical trials. ${ }^{3,5,6,22}$ However, it remains unclear how and why the involvement of one therapeutic approach could affect the effect of another. Our study provides evidence that CpG-based immunotherapy can upregulate the production of B-cell-derived TNFa and thereby negatively influence antitumor responses to BRAF inhibitors. Given the established role of TNFa in suppressing antitumor activity of mitogen-activated protein kinase inhibitors, ${ }^{27-29}$ it is highly likely that CpG treatment can also result in enhanced resistance to mitogen-activated protein kinase inhibitors. This implicates the adverse impact that CpGbased immune treatment may have when combined with mitogen-activated protein kinases pathway inhibition in general.

Taken together, our study demonstrated that CpG treatment can impair the antitumor activity of BRAF inhibitors by enhancing the production of TNFa by B cells. More importantly, our results strongly suggest that precautions must be implemented when designing combinatorial approaches for cancer treatment, because distinct regimens, despite their respective therapeutic benefit, may together provide antagonistic, rather than synergistic, clinical outcomes.

\section{CONFLICT OF INTEREST}

The authors declare no conflict of interest.

\section{ACKNOWLEDGEMENTS}

We thank Dr Hussein Tawbi for constructive comments on the manuscript. The research was supported in part by the following National Cancer Institute grants: R01CA116206 (to PH), P01CA128913 (to PH), R01CA187076 (to PH and MD), P50CA093459 and P30CA016672 (MDACC Melanoma SPORE and MDACC CCSG for JAM and Flow facility, respectively). By the Cancer Prevention and Research Institute of Texas (CPRIT RP140106 to JAM) and by philanthropic contributions to MDACC Melanoma Moon Shot Program; Melanoma Research Alliance Team Science Award; Dr Miriam and Sheldon G Adelson Medical Research Foundation; Aim at Melanoma Foundation, Miriam and Jim Mulva research funds; Jurgen Sager \& Transocean Melanoma Research Fund; El Paso Foundation for Melanoma Research; Gillson
Logenbaugh Foundation. We thank Cherie Butts from Biogen for providing antimouse CD20 antibody.

\section{AUTHOR CONTRIBUTIONS}

$\mathrm{PH}$ and WP conceived and supervised the research study. $\mathrm{LH}, \mathrm{ZW}, \mathrm{CL}, \mathrm{CX}, \mathrm{RM}$, JM, HK and WP conducted experiments, acquired and analyzed data. LH, ZW and WP wrote the manuscript.

\section{REFERENCES}

1 Kim T, Amaria RN, Spencer C, Reuben A, Cooper ZA, Wargo JA. Combining targeted therapy and immune checkpoint inhibitors in the treatment of metastatic melanoma. Cancer Biol Med 2014; 11: 237-246.

2 Hu-Lieskovan S, Robert L, Homet Moreno B, Ribas A. Combining targeted therapy with immunotherapy in BRAF-mutant melanoma: promise and challenges. J Clin Oncol 2014; 32: 2248-2254.

3 Cooper ZA, Juneja VR, Sage PT, Frederick DT, Piris A, Mitra D et al. Response to BRAF inhibition in melanoma is enhanced when combined with immune checkpoint blockade. Cancer Immunol Res 2014; 2: 643-654.

4 Hailemichael Y, Dai Z, Jaffarzad N, Ye Y, Medina MA, Huang XF et al. Persistent antigen at vaccination sites induces tumor-specific CD8(+) T cell sequestration, dysfunction and deletion. Nat Med 2013; 19: 465-472.

5 Hu-Lieskovan S, Mok S, Homet Moreno B, Tsoi J, Robert L, Goedert L et al. Improved antitumor activity of immunotherapy with BRAF and MEK inhibitors in BRAF(V600E) melanoma. Sci Transl Med 2015; 7: 279 ra41.

6 Koya RC, Mok S, Otte N, Blacketor KJ, Comin-Anduix B, Tumeh PC et al. BRAF inhibitor vemurafenib improves the antitumor activity of adoptive cell immunotherapy. Cancer Res 2012; 72: 3928-3937.

7 Mehta NK, Moynihan KD, Irvine DJ. Engineering new approaches to cancer vaccines. Cancer Immunol Res 2015; 3: 836-843.

8 Melief CJ, van Hall T, Arens R, Ossendorp F, van der Burg SH. Therapeutic cancer vaccines. J Clin Invest 2015; 125: 3401-3412.

9 Bode C, Zhao G, Steinhagen F, Kinjo T, Klinman DM. CpG DNA as a vaccine adjuvant. Expert Rev Vaccines 2011; 10: 499-511.

10 Weiner GJ, Liu HM, Wooldridge JE, Dahle CE, Krieg AM. Immunostimulatory oligodeoxynucleotides containing the CpG motif are effective as immune adjuvants in tumor antigen immunization. Proc Natl Acad Sci USA 1997; 94: 10833-10837.

11 Bauer S, Wagner H. Bacterial CpG-DNA licenses TLR9. Curr Top Microbiol Immunol 2002; 270: 145-154.

12 Rothenfusser S, Tuma E, Endres S, Hartmann G. Plasmacytoid dendritic cells: the key to CpG. Hum Immunol 2002; 63: 1111-1119.

13 Davila E, Kennedy R, Celis E. Generation of antitumor immunity by cytotoxic T lymphocyte epitope peptide vaccination, CpG-oligodeoxynucleotide adjuvant, and CTLA-4 blockade. Cancer Res 2003; 63: 3281-3288.

14 Jerome V, Graser A, Muller R, Kontermann RE, Konur A. Cytotoxic T lymphocytes responding to low dose TRP2 antigen are induced against B16 melanoma by liposome-encapsulated TRP2 peptide and CpG DNA adjuvant. J Immunother 2006; 29: 294-305.

15 Vollmer J, Krieg AM. Immunotherapeutic applications of CpG oligodeoxynucleotide TLR9 agonists. Adv Drug Deliv Rev 2009; 61: 195-204.

16 Scheiermann J, Klinman DM. Clinical evaluation of CpG oligonucleotides as adjuvants for vaccines targeting infectious diseases and cancer. Vaccine 2014; 32: 6377-6389.

17 Speiser DE, Lienard D, Rufer N, Rubio-Godoy V, Rimoldi D, Lejeune F et al. Rapid and strong human CD8+ T cell responses to vaccination with peptide, IFA, and CpG oligodeoxynucleotide 7909. J Clin Invest 2005; 115: 739-746.

18 Liu C, Lou Y, Lizee G, Qin H, Liu S, Rabinovich B et al. Plasmacytoid dendritic cells induce NK cell-dependent, tumor antigen-specific $\mathrm{T}$ cell cross-priming and tumor regression in mice. J Clin Invest 2008; 118: 1165-1175.

19 Lou YY, Liu CW, Lizee G, Peng WY, Xu CY, Ye Y et al. Antitumor activity mediated by CpG: the route of administration is critical. J Immunother 2011; 34: 279-288.

20 Flaherty KT, Puzanov I, Kim KB, Ribas A, McArthur GA, Sosman JA et al. Inhibition of mutated, activated BRAF in metastatic melanoma. N Engl J Med 2010; 363: 809-819.

21 Pashenkov M, Goess G, Wagner C, Hormann M, Jandl T, Moser A et al. Phase II trial of a toll-like receptor 9-activating oligonucleotide in patients with metastatic melanoma. J Clin Oncol 2006; 24: 5716-5724.

22 Ribas A, Hodi FS, Callahan M, Konto C, Wolchok J. Hepatotoxicity with combination of vemurafenib and ipilimumab. N Engl J Med 2013; 368: 1365-1366.

23 Dankort D, Curley DP, Cartlidge RA, Nelson B, Karnezis AN, Damsky WE Jr et al. Braf(V600E) cooperates with Pten loss to induce metastatic melanoma. Nat Genet 2009; 41: 544-552. 
4086

24 Shirota H, Tross D, Klinman DM. CpG oligonucleotides as cancer vaccine adjuvants. Vaccines 2015; 3: 390-407.

25 Hanten JA, Vasilakos JP, Riter CL, Neys L, Lipson KE, Alkan SS et al. Comparison of human B cell activation by TLR7 and TLR9 agonists. BMC Immunol 2008; 9: 39.

26 Avalos AM, Latz E, Mousseau B, Christensen SR, Shlomchik MJ, Lund F et al. Differential cytokine production and bystander activation of autoreactive $B$ cells in response to CpG-A and CpG-B oligonucleotides. J Immunoly 2009; 183: 6262-6268.

27 Smith MP, Sanchez-Laorden B, O'Brien K, Brunton H, Ferguson J, Young $\mathrm{H}$ et al. The immune microenvironment confers resistance to MAPK pathway inhibitors through macrophage-derived TNFalpha. Cancer Discov 2014; 4: 1214-1229.

28 Menon DR, Wels C, Bonyadi Rad E, Joshi S, Knausz H, Lade-Keller J et al. TGF-beta1 and TNF-alpha differentially regulate Twist 1 mediated resistance towards BRAF/ MEK inhibition in melanoma. Pigment Cell Melanoma Res 2013; 26: 912-916.

29 Gray-Schopfer VC, Karasarides M, Hayward R, Marais R. Tumor necrosis factoralpha blocks apoptosis in melanoma cells when BRAF signaling is inhibited. Cancer Res 2007; 67: 122-129.

30 Shirota H, Klinman DM. Recent progress concerning CpG DNA and its use as a vaccine adjuvant. Expert Rev Vaccines 2014; 13: 299-312.

31 Perricone MA, Smith KA, Claussen KA, Plog MS, Hempel DM, Roberts BL et al. Enhanced efficacy of melanoma vaccines in the absence of $B$ lymphocytes. $J$ Immunother 2004; 27: 273-281.

32 Kim S, Fridlender ZG, Dunn R, Kehry MR, Kapoor V, Blouin A et al. B-cell depletion using an anti-CD20 antibody augments antitumor immune responses and immunotherapy in nonhematopoetic murine tumor models. J Immunother 2008; 31: 446-457.

33 Tadmor T, Zhang Y, Cho HM, Podack ER, Rosenblatt JD. The absence of B lymphocytes reduces the number and function of T-regulatory cells and enhances the anti-tumor response in a murine tumor model. Cancer Immunol Immunother 2011; 60: 609-619.

34 Peng W, Ye Y, Rabinovich BA, Liu C, Lou Y, Zhang M et al. Transduction of tumorspecific $T$ cells with CXCR2 chemokine receptor improves migration to tumor and antitumor immune responses. Clin Cancer Res 2010; 16: 5458-5468.

35 Liu C, Peng W, Xu C, Lou Y, Zhang M, Wargo JA et al. BRAF inhibition increases tumor infiltration by $T$ cells and enhances the antitumor activity of adoptive immunotherapy in mice. Clin Cancer Res 2013; 19: 393-403.

(i) (2) This work is licensed under a Creative Commons AttributionBY NC SA NonCommercial-ShareAlike 4.0 International License. The images or other third party material in this article are included in the article's Creative Commons license, unless indicated otherwise in the credit line; if the material is not included under the Creative Commons license, users will need to obtain permission from the license holder to reproduce the material. To view a copy of this license, visit http:// creativecommons.org/licenses/by-nc-sa/4.0/

(c) The Author(s) 2017

Supplementary Information accompanies this paper on the Oncogene website (http://www.nature.com/onc) 